\title{
Diacronie
}

Studi di Storia Contemporanea

$\mathrm{N}^{\circ} 24,4 \mid 2015$

Le dittature militari: fisionomia ed eredità politica

\section{Il Derg, Siad Barre e l'Ogaden}

Due diverse concezioni di dittatura militare e il loro scontro in una guerra per procura

\section{Francesco Maria Mengo}

\section{(2) OpenEdition \\ 1 Journals}

\section{Edizione digitale}

URL: http://journals.openedition.org/diacronie/3832

DOI: $10.4000 /$ diacronie.3832

ISSN: 2038-0925

Editore

Association culturelle Diacronie

Notizia bibliografica digitale

Francesco Maria Mengo, « II Derg, Siad Barre e l'Ogaden », Diacronie [Online], № 24, 4 | 2015, documento 16, Messo online il 29 décembre 2015, consultato il 19 avril 2019. URL : http:// journals.openedition.org/diacronie/3832 ; DOI : 10.4000/diacronie.3832 


\section{Diacronie}

\section{6/}

\section{Il Derg, Siad Barre e l'Ogaden}

\section{Due diverse concezioni di dittatura militare e il loro scontro in una guerra per procura}

Francesco Maria MENGO *

L'obiettivo dell'articolo è mostrare le diverse concezioni di organizzazione e gestione del potere di due dittature militari dell'Africa Orientale: il governo del Derg (Etiopia, 1974-87) e l'autocrazia di Siad Barre (Somalia, 1969-91). Entrambi i governi promossero politiche riconducibili al marxismo e rimasero nell'ambito diplomatico del terzomondismo. Nella guerra dell'Ogaden (1977-78) gli USA appoggiarono economicamente e militarmente la Somalia; il Secondo Mondo fornì a sua volta aiuti militari al Derg. Si mostrerà nondimeno come la visione della guerra dell'Ogaden come un semplice scontro per procura possa essere limitativa.

\section{Introduzione}

$\mathrm{I}$

n questo articolo saranno esaminate le concezioni di organizzazione e gestione del potere in due dittature militari dell'Africa Orientale: il governo del Derg (amarico per "Consiglio") in Etiopia, al potere tra il 1974 e il 1987, e l'autocrazia di Siad Barre in Somalia, durata dal 1969 al 1991. Analizzeremo le differenti anatomie politiche e i differenti strumenti di gestione del potere delle due dittature, nell'ottica di una comparazione di stampo storico-politologico.

Il Derg era un consiglio militare con dinamiche decisionali collegiali: ha sempre agito in forma di giunta, nonostante le tendenze all'accentramento del potere di alcuni suoi appartenenti, su tutti quel Mengistu al cui nome la dittatura del Derg è comunemente associata, andando a creare un immaginario che tende a non dare il giusto peso alla collegialità dell'organismo. I componenti del Derg erano 
ideologicamente vicini al marxismo-leninismo, benché il paese abbia continuato a rimanere nel Movimento dei Non Allineati, e il Derg accordò alle linee guida del socialismo sovietico le proprie scelte politiche, strutturando a tal fine l'apparato ideologico dell'Ye-Itiopia Hibret's'bawinet ("Vita comunitaria dell’Etiopia" in amarico).

Quello di Maxamed Siad Barre, al contrario, fu un governo dalle spiccate tendenze autocratiche, visibili già dalla presa del potere. Il marxismo-leninismo ispirò nei primi tempi il governo di Siad Barre, ma egli ne mitigò gradualmente l'applicazione con la teorizzazione politologica di un socialismo scientifico che includeva l'Islam come componente organica. Nell'ambito diplomatico anche la Somalia di Siad Barre rimase nell'alveo multiforme del terzomondismo, non chiudendosi a nessuna opportunità di alleanza politica ed economica.

Stati Uniti e mondo arabo appoggiarono la Somalia nella guerra dell'Ogaden, tra il 1977 e il 1978, fornendole aiuti militari ed economici a vario titolo; la parte di mondo gravitante nell'orbita politica dell'Unione Sovietica, a sua volta, non mancò di sostenere militarmente l'Etiopia del Derg. Nella guerra dell'Ogaden si estrinsecarono tensioni a vari livelli: fu uno scontro tra dittature aventi diverse strutture di autorità e diverse basi ideologiche di autogiustificazione e azione politica. Fu uno scontro per le risorse dell'Ogaden e uno scontro per il predominio geopolitico ed economico nella regione dell'Africa Orientale. Fu la svolta su una più larga scala di violenza di tensioni che proseguivano da diversi anni. Fu peraltro uno scontro che, essendo tra due entità politiche nelle quali una cultura politica nazionalista aveva avuto modo di affermarsi in quanto medium legittimante dei regimi, venne ampiamente fomentato dalle istanze nazionaliste di ambo le parti. La guerra dell'Ogaden viene però soprattutto ricordata come una guerra conto terzi, nel contesto della guerra fredda e delle sue estrinsecazioni di scontro ${ }^{1}$.

L'articolo si concluderà con un riepilogo delle similitudini e un'anamnesi delle più evidenti differenze politologiche tra le dittature del Derg e di Siad Barre.

Le fonti qui trattate saranno perlopiù di natura editoriale: non è possibile, ad esempio, risalire a documentazione primaria prodotta dal Derg e concernente la sua struttura e la sua attività, qualora esistesse. Le nostre principali fonti riguardo la rivoluzione del Derg saranno The Ethiopian Revolution dello storico etiope Andargachew Tiruneh² e

${ }^{1}$ JACKSON, Donna R., Jimmy Carter and the Horn of Africa: Cold War Policy in Ethiopia and Somalia, Jefferson, McFarland \& Company, 2007, pp. 85-87; CLAPHAM, Cristopher, International Relations in Africa after the Cold War, in HALE, William, KIENLE, Eberhard (edited by), After the Cold War: Security and Democracy in Africa and Asia, London, Tauris Academic Studies, 1997, p. 101; DOCKRILL, Michael L., HOPKINS, Michael F., The Cold War, 1945-1991, Basingstoke, Palgrave MacMillan, 2006, pp. 127-128.

2 TIRUNEH, Andargachew, The Ethiopian revolution 1974-1987. A transformation from an 
Revolutionary Ethiopia di Edmond Keller3. Per la Somalia di Siad Barre, due ottime opere da cui partire sono la monografia dello storico francese Daniel Compagnon 4 e l'opera biografica che Mohammed Ibrahim Shire ha dedicato al dittatore5.

\section{Il Derg}

$\mathrm{Al}$ momento della rivoluzione del Derg, l'impero etiope di Haile Selassie era in uno dei momenti di maggiore delegittimazione della sua storia. Nel 1960 era già fallito un tentativo di colpo di stato militare; nel nord, intanto, la questione nazionale eritrea e le mobilitazioni indipendentiste facevano sentire il loro peso, venendo represse con un grande dispendio di energie e risorse. A tutto ciò va aggiunta l'impotenza governativa di fronte alle due carestie del Welo e del Tigré, che duravano dall'inizio del decennio.

Nel gennaio 1974 iniziarono ammutinamenti diffusi nell'esercito etiope. Non va dimenticato come, in uno stato povero, molto popoloso e con un enorme apparato militare come l'Etiopia del tempo, l'esercito avesse una funzione di ammortizzatore sociale: i militari provenivano spesso dalle stesse classi sociali che stavano soffrendo la fame. Sembra inoltre che vari militari in rivolta stessero avendo contatti politici con intellettuali marxisti ${ }^{6}$. Alla mobilitazione dell'esercito si aggiunse subito quella di studenti e operai, che presero per giorni le strade di Addis Abeba e delle altre maggiori città etiopi. A giugno si formò un collegio di sottufficiali e ufficiali di basso grado, uniti dall'identificazione ideologica marxista e dall'opposizione al regime imperiale. Era nato il Derg: il suo presidente provvisorio, eletto in forma collegiale, era il maggiore Haile Mariam Mengistu, e il suo portavoce il maggiore Atnafu Abate.

Il Derg raccolse un ampio consenso tra le truppe già ammutinate, e tramite il loro appoggio iniziò ad arrestare di sua iniziativa esponenti dell'aristocrazia terriera e politica, insieme ai militari rimasti fedeli all'impero. Ad agosto il Derg dichiarò sciolte le camere, si impossessò della Banca Nazionale e nazionalizzò le proprietà private imperiali; il 12 settembre dichiarò decaduto Haile Selassie, imprigionandolo.

Il Derg prese la guida del governo il 15 settembre, senza tuttavia uscire dalla

aristocratic to a totalitarian autocracy, Cambridge, Cambridge University Press, 1993.

3 KELLER, Edmond J., Revolutionary Ethiopia. From Empire To People's Republic, Bloomington, Indiana University Press, 1991.

4 COMPAGNON, Daniel, Ressources politiques, régulation autoritaire et domination personnelle en Somalie: Le régime de Siyaad Barre, 1969-1991, Pau, Université de Pau et des Pays de l'Adour, 1995.

5 SHIRE, Mohammed Ibrahim, Somali President Mohammed Siad Barre. His Life and Legacy, Leicester, Cirfe, 2011.

6 CLAPHAM, Christopher, Transformation and Continuity in Revolutionary Ethiopia, Cambridge, Cambridge University Press, 1988, p. 43. 
condizione di chiusura in cui era nato: l'affiliazione dei 106 (resi pubblici come 1207) membri iniziali continuava a rimanere anonima, e l'appartenenza al Derg era pubblicamente segreta ${ }^{8}$. Il Derg nasceva, insomma, come un'istituzione incomunicante con l'esterno: una sorta di sistema chiuso di oligarchia militare. I 106 membri iniziali, peraltro, andarono a diminuire di numero nel corso degli anni, ma non si sviluppò alcun processo di ricambio al suo interno?.

Cionondimeno, le decisioni venivano prese in forma collegiale: le mozioni proposte venivano votate e la posizione delle cariche esterne di presidente e portavoce valeva quanto ognuna delle altre, essendo come queste espressa nel voto.

Tali due cariche, comunque, non mancarono di fare gola, avendo la carica di presidente il diritto di decisione finale sull'approvazione delle mozioni mediante firma e rappresentando le due cariche le uniche forme di contatto del Derg con la popolazione.

Fu per questo motivo, ad esempio, che Mengistu ricercò la presidenza, persa dopo aver guidato ad interim il Derg a favore dell'allora tenente Tafari Benti, eletto collegialmente nel novembre 1974 in quanto figura moderata che avrebbe potuto fungere da calmiere delle diverse istanze interne al Consiglio ${ }^{10}$. Mengistu, pur muovendosi all'interno di una dinamica decisionale consiliare, intendeva riottenere il suo ruolo di presidente, e per far ciò sarebbe stato necessario l'ottenimento di una maggioranza all'interno del Derg. E, talvolta, nuove maggioranze si ottengono anche con l'eliminazione di quelle esistenti. Il 3 febbraio 1977 la riunione plenaria del Derg venne sconvolta da una sparatoria. Morirono 58 membri: tra questi lo stesso Benti, insieme a chiunque avesse in passato mostrato opposizione a Mengistu. Questi venne rinominato collegialmente presidente, all'unanimità, e rimase tale fino allo scioglimento del Derg'11.

Il $1^{\circ}$ novembre $1974^{12}$ venne emanato il documento programmatico Ethiopia Tikdem (in amarico "Prima l'Etiopia"), il quale sintetizzava i principi della nuova filosofia politica del Hibret's'bawinet. Nell'Ethiopia Tikdem un riferimento politico al marxismo-leninismo sovietico non venne apertamente dichiarato: il documento anzi,

\footnotetext{
7 TIRUNEH, Andargachew, op. cit., p. 64.

8 KELLER, Edmond J., op. cit., p. 192.

9 TIRUNEH, Andargachew, op. cit., pp. 164-166.

1o OTTAWAY, Marina, OTTAWAY, David, Ethiopia. Empire in Revolution, New York City, Africana Publishing Company, 1978, p. 134.

11 Ibidem, p. 143; KELLER, Edmond J., op. cit., p. 204.

${ }_{12}^{12}$ HAILE-SELASSIE, Teferra, The Ethiopian Revolution 1974-1991. From A Monarchical Autocracy To A Military Oligarchy, Abingdon, Routledge, 2011, p. 135. Edmond Keller data però la pubblica emanazione dell'Ethiopia Tikdem al successivo 20 dicembre. KELLER, Edmond J., op. cit., p. 193.
} 
come già intuibile dal nome, evocava immaginari nazionalisti ${ }^{13}$, e le ideologie provenienti da contesti culturali altri rispetto a quello etiope sembravano venir guardate con disdegno ${ }^{14}$. Forte era anche l'enfasi sull'azione comune delle diverse «affiliazioni etniche» dell'Etiopia: si tese a propagandare l'unità interna delle diverse componenti, contrapponendole a fattori esterni di alterità. Altri punti programmatici, comunque, evocavano i modelli sovietici di statalizzazione e suddivisione dei mezzi di produzione e delle ricchezze.

Avendo l'Etiopia un'economia basata largamente sulla produzione agricola di sussistenza ed essendo state le sue terre in massima parte latifondo di proprietà nobiliare, la riforma agraria fu uno dei punti chiave per la costruzione di un sistema di rapporti socioeconomici socialista: nel marzo 1975 il Derg nazionalizzò tutti i terreni coltivabili, li suddivise per quantità stabilite sulla base dei nuclei famigliari e vietò l'affitto agli assegnatari. Banche, assicurazioni e grandi imprese erano già state nazionalizzate tra gennaio e febbraio ${ }^{15}$.

L'Unione Sovietica continuò, nei primi tempi, a corteggiare la Somalia di Siad Barre. Nel giugno 1976, però, venne ufficialmente decretata dal Congresso la fine del sostegno militare statunitense all'Etiopia del Derg marxista ${ }^{16}$; davanti a tale situazione, l'URSS iniziò ad approfittare della vicinanza ideologica del Derg, e iniziò a sostenere militarmente - oltre che politicamente - l'Etiopia di Mengistu, fornendole armamenti. Nel dicembre 1976 Etiopia e Unione Sovietica firmarono un accordo per un invio di armamenti dal valore complessivo di circa 100 milioni di dollari ${ }^{17}$. Dal 1975, intanto, i più alti ranghi dell'esercito etiope si stavano sottoponendo ad addestramenti militari in Unione Sovietica e in paesi a essa alleati, come la Germania Est, la Bulgaria, la Cecoslovacchia e Cuba; dal 1977, in coincidenza con l'invasione somala dell'Ogaden, la possibilità di beneficiare di tali addestramenti venne estesa a tutto il personale militare ${ }^{18}$.

Vista la fine del sostegno statunitense, viste le ambiguità somale - di cui parleremo più nel dettaglio - e visto che Mengistu cercò esplicitamente una più stretta alleanza con

\footnotetext{
${ }^{13}$ HAILE-SELASSIE, Teferra, op. cit., pp. 135-136.

14 KELLER, Edmond J., op. cit., p. 193.

15 OFCANSKY, Thomas P., BERRY, LaVerle Bennette, Ethiopia: a country study, Washington, D.C., Library of Congress. Federal Research Division, 1993, p. 57.

16 US Congressional Record, 94th Congress, H.R.13680 - An Act to amend the Foreign Assistance Act of 1961 and the Foreign Military Sales Act, and for other purposes. Conference report filed in House, H. Rept. 94-1272, 06/16/1976.

${ }_{17}$ JACKSON, Donna R., op. cit., p. 49.

${ }_{18}$ CLAPHAM, Christopher, op. cit., pp. 67, 224.
} 
l'Unione Sovietica, questa non ebbe problemi a iniziare a fornire sostegno politico, militare ed economico all'Etiopia ${ }^{19}$.

\section{Siad Barre}

Nel 1969 il presidente della Repubblica somalo Abdirashid Ali Shermarke venne ucciso da una guardia del corpo. Il Primo ministro Maxamed Hajj Ibrahim Igaal spinse l'Assemblea Nazionale a nominare presidente della Repubblica Muqtar Maxamed Husein, altro esponente di spicco del partito di governo, la Lega dei Giovani Somali. Il 21 ottobre 1969 unità dell'esercito presero il controllo della città di Mogadiscio, irrompendo anche nell'Assemblea; la polizia, in collaborazione con i militari insorti, non intervenne.

Se il marxismo-leninismo aveva una grande diffusione entro l'esercito somalo, una gran parte delle responsabilità al riguardo fu del suo comandante in capo Maxamed Siad Barre, che aveva piena autorità nella gestione di vari aspetti della logistica militare, tra i quali anche le operazioni di formazione dei sottoposti. Peraltro, dal momento che l'esercito somalo sin dall'indipendenza aveva iniziato a inviare i propri ufficiali ad addestrarsi nell'Unione Sovietica, molti di essi avevano avuto modo di scoprire il marxismo-leninismo e avvicinarvisi politicamente; tra questi lo stesso Siad Barre $^{20}$.

Il corpo di ufficiali che compì il golpe nell'ottobre 1969 si era denominato Consiglio Rivoluzionario Supremo, e inizialmente sembrò agire con la stessa collegialità con cui in seguito avrebbe agito il Derg. Fu presa a un livello ufficialmente collegiale, ad esempio, la decisione immediata di mettere al bando i partiti, sciogliere l'Assemblea Nazionale e sospendere la Costituzione ${ }^{21}$.

Le tendenze autocratiche di Siad Barre, comunque, non tardarono a emergere, facilitate dalla struttura istituzionale che, avendogli conferito il ruolo di presidente della Repubblica con le connesse prerogative esecutive, non avrebbe potuto limitarle. All'interno degli organismi governativi ebbe modo però di emergere un'altra figura dalle personali tendenze leaderistiche, quella del vicepresidente e ministro degli Interni Jaama Ali Qoorshel. Nel 1970 venne portato alla luce un presunto tentativo di

19 THOMPSON, William J., The Soviet Union under Brezhnev New York City, Routledge, 2014, p. 54 .

${ }^{20}$ PATMAN, Robert G., The Soviet Union in the Horn of Africa. The diplomacy of intervention and disengagement, New York City, Cambridge University Press, 1990, p. 96.

${ }^{21}$ CHAPIN METZ, Helen, Somalia: a country study, Washington, D.C., Library of Congress. Federal Research Division, 1993, p. 46. 
controgolpe da lui orchestrato ${ }^{22}$; in seguito a ciò, il vicepresidente e altri membri del Consiglio a lui vicini vennero arrestati. Siad Barre non aveva più opposizione all’interno delle componenti di governo.

Nel 1976 Siad Barre sciolse il Consiglio Rivoluzionario Supremo per trasferire il controllo politico del regime nelle mani di un partito, fondando lo XHKS (Xisbiga Hantiwadaagga Kacaanka Soomaalyieed, Partito Socialista Rivoluzionario Somalo), di cui il generale si trovò a ricoprire le cariche di presidente e segretario. L'XHKS ebbe anche una struttura organizzativa elementare: venne creato un Comitato Centrale con un Politburo di cinque membri, ma i componenti di tale ultimo ramo erano tutti militari già golpisti, e più in generale all'interno della struttura del partito l'appoggio a Siad Barre era totale ${ }^{23}$. Ciò permise al nuovo capo di Stato di portare avanti la pubblica legittimazione del proprio potere attraverso mezzi autocratici di culto della personalità: promuovendo il pubblico riferimento alla sua persona come Guulwadde ("Comandante vittorioso") si fece rappresentare come il nuovo e lungimirante leader politico che aveva saputo unire in modo rivoluzionario Islam e socialismo, fece raccogliere i suoi proclami in un libretto bianco e azzurro (i colori della bandiera somala) che fece distribuire nelle scuole e nei luoghi di lavoro e la sua faccia iniziò a comparire al fianco di quelle di Marx e Lenin in enormi ritratti posti a ogni angolo della capitale e delle maggiori città del paese.

L'XHKS si definiva socialista sin dalla sua denominazione; al contrario del Derg, però, neanche nella pratica politica era riscontrabile un'ortodossia al marxismoleninismo. L'applicazione di quello che lo stesso Siad Barre definì "socialismo scientifico" ebbe diverse influenze provenienti da ben altre istanze, prima su tutte l'Islam.

Nel primo anniversario della rivoluzione, Siad Barre enunciò in un discorso alla nazione le basi di quello che definì il «socialismo scientifico» somalo. Dopo aver dichiarato ufficialmente per la prima volta la Somalia uno «Stato socialista», il presidente delineò le tre macrocategorie ideologiche da cui la nuova ideologia del socialismo scientifico somalo doveva, nei suoi piani, derivare: il marxismo-leninismo ortodosso, l'Islam e l'«autosufficienza somala». Quest'ultimo aspetto, non meglio specificato se non nella necessità di «preservazione delle tradizioni», fungeva evidentemente da collante consensuale nei confronti della popolazione, consentendogli

${ }^{22}$ OTTAWAY, Marina, Soviet and American Influence in the Horn of Africa, Westport, Praeger, 1982, p. 64.

23 JACKSON, Robert H., ROSBERG, Carl Gustav, Personal Rule in Black Africa. Prince, Autocrat, Prophet, Tyrant, Berkeley, University of California Press, 1982, p. 196. 
di portare avanti proclami basati sul più classico nazionalismo terzomondista ${ }^{24}$.

L'applicazione pratica dei principi di regolamentazione economica del socialismo, in realtà, andò a colpire esclusivamente le entità economiche più organicamente strutturate e aventi una maggiore caratterizzazione di formalità, come banche, assicurazioni e altre categorie di grandi imprese operanti coram populo nei centri maggiori; i rapporti di produzione e di proprietà non vennero sconvolti al di fuori della più grande economia capitalista. Come Daniel Compagnon fa notare ${ }^{25}$, pur se nel contesto di esautorazione delle autorità religiose a livello locale, Siad Barre non tentò completamente di scavalcare le differenti stratificazioni di autorità sociale già presenti, a partire dai meccanismi di clan che regolavano capillarmente i rapporti di potere a livello locale, lasciando loro spazi di manovra specialmente nel campo del commercio e della proprietà privata. Il potere sociale che tali entità si erano costruite passava anche dal piano economico: in una società di agricoltura di sussistenza senza rapporti di potere feudali quale era quella somala una grande fonte di guadagno era ad esempio il commercio di bestiame. A tali entità venne consentito di portare avanti le proprie attività, senza che lo Stato si addentrasse eccessivamente nella loro regolamentazione, in modo tale da garantirsi la fedeltà e la non opposizione di figure locali di autorevolezza - ancor prima che di autorità - attraverso meccanismi di fatto clientelari.

Nel maggio 1970, intanto, il nuovo governo aveva nazionalizzato le aziende straniere presenti, dalle banche alle assicurazioni alle diramazioni delle petrolifere Shell e Agip. Susseguente fu l'espulsione dal paese del personale militare dell'ambasciata statunitense di Mogadiscio; in seguito a tali misure, gli Stati Uniti bloccarono il loro programma di aiuti annuali alla Somalia. Di converso, l'Unione Sovietica aveva accolto positivamente sin da subito l'arrivo di Siad Barre al potere, e già nei primi mesi del nuovo governo iniziò a far ospitare in territorio somalo propri mezzi militari e a fornire aiuti economici di vario genere. Ciononostante, in seguito alla proclamazione del socialismo scientifico l'Unione Sovietica pose in questione l'ortodossia marxista delle dichiarazioni di Siad Barre, non presente e di fatto non voluta. Un'altra occasione di freddezza di Siad Barre nei confronti dell'URSS fu il rifiuto di firmare un trattato bilaterale di amicizia nell'occasione della visita di stato dell'allora

24 CHAPIN METZ, Helen, Somalia: a country study, Washington, D.C., Library of Congress. Federal Research Division, 1993, p. 42; GEBREWOLD, Belachew, Anatomy of Violence. Understanding the Systems of Conflict and Violence in Africa, Farnham, Ashgate, 2009, pp. 130-131.

${ }_{25}$ COMPAGNON, Daniel, «State-sponsored violence and conflict under Mahamed Siyad Barre: the emergence of path dependent patterns of violence», in World Peace Foundation, URL: $<$ https://sites.tufts.edu/reinventingpeace/2013/10/22/state-sponsored-violence-and-conflictunder-mahamed-siyad-barre-the-emergence-of-path-dependent-patterns-ofviolence/\#_ftnref5> [consultato il 15 novembre 2015]. 
ministro della Difesa sovietico Andreij Grečko, nel febbraio 1972. Siad Barre, rifiutando di entrare in una dinamica diplomatica di alleanza eccessivamente stretta con l'Unione Sovietica, cercò di non alienarsi le simpatie dei paesi arabi conservatori e vicini al blocco occidentale, ai quali cercò di mantenersi vicino attraverso l'insistenza sulla comune affiliazione religiosa diffusa, l'Islam sunnita ${ }^{26}$.

L'aderenza ai principi di regolamentazione della vita pubblica dettati dai codici coranici fu infatti un altro dei capisaldi teorici del socialismo scientifico di Siad Barre. È necessario tenere in mente, oltre alle ragioni di politica estera, come in Somalia l'Islam avesse una diffusione tale da essere un mezzo di leva sul consenso della popolazione ben più efficace di quanto potesse esserlo il marxismo-leninismo. Il tentativo di Siad Barre di accostare ed equiparare a un livello teorico principi islamici e marxismoleninismo ateo provocò, come comprensibile, tensioni con le autorità teologiche del paese, fin da quando nella presentazione del socialismo scientifico equiparò i due sistemi di pensiero sostenendo che entrambi promuovessero «giustizia, uguaglianza e miglioramento delle vite delle persone». Nel 1975, a complicare le tensioni tra potere spirituale e autorità statale, intervennero gli arresti e le esecuzioni di dieci personalità religiose con l'accusa di aver invitato i fedeli all'opposizione ${ }^{27}$.

In effetti, nonostante le ripetute professioni di fede nell'Islam e nei suoi principi regolatori della vita sociale da parte di Siad Barre, sembra che egli abbia teso più che altro - nel quadro dei rapporti tra Stato ed entità religiose - a minare l'autorità di regolamentazione della vita sociale somala delle figure religiose. Il progetto di alfabetizzazione della popolazione ebbe ad esempio contraccolpi sulle scuole coraniche, che videro diminuire drasticamente i propri alunni. Gli wadaddo, figure religiose aventi autorità civile nei villaggi, vennero inoltre esautorati di ogni competenza legale quando il governo degli enti locali venne affidato a funzionari statali28.

L'attenzione di Siad Barre all'Islam si sviluppò soprattutto sul piano diplomatico, attraverso la costruzione di rapporti di collaborazione economica e militare con la monarchia saudita, politicamente vicina agli Stati Uniti ${ }^{29}$. I rapporti di collaborazione tra la Somalia e l'Unione Sovietica, in seguito alle frizioni ideologiche di cui sopra, in effetti non finirono: nel luglio 1972, ad esempio, l'URSS iniziò a inviare - oltre ai

${ }^{26}$ PATMAN, Robert G., op. cit., pp. 93-96, 116-118.

${ }^{27}$ SHEIKH HASAN, Mohamed Rashid, ROBLEH, Salada M., Islamic Revival and Education in Somalia, in DAUN, Holger, WALFORD, Geoffrey (a cura di), Educational Strategies among Muslims in the Context of Globalization. Some National Case Studies, Leiden, Brill, 2004, p. 150.

${ }^{28}$ FITZGERALD, Nina J., Somalia. Issues, History and Bibliography, New York City, Nova, 2002, p. 23.

29 QUANDT, William B., Saudi Arabia in the 1980s. Foreign Policy, Security and Oil, Washington D.C., The Brookings Institution, 1981, p. 45. 
contributi economici sopra ricordati - anche i primi aiuti militari al paese del Corno d'Africa, che già fungeva da base di appoggio per i mezzi navali e aerei sovietici3o. Nondimeno, per avvicinarsi al mondo arabo facente più stretto riferimento al blocco occidentale, nel 1974 Siad Barre fece entrare la Somalia anche nella Lega Araba.

La Somalia faceva parte del Movimento dei Paesi Non Allineati già dal 1961. Il terzomondismo rappresentava per la Somalia, come per diversi altri paesi, un'occasione per una presa di posizione equidistante dai due blocchi e mirata alla ricerca di buoni rapporti tanto con la sfera sovietica quanto con paesi facenti parte del blocco politico e socioeconomico liberale, nello specifico l'Arabia Saudita e i paesi islamici politicamente orbitanti al suo intorno.

Nell'ottobre 1974, dopo l'ingresso nella Lega Araba, la Somalia concesse addirittura agli Stati Uniti l'utilizzo come base militare navale del porto di Kismayu, nel Sud del paese, continuando ad avere a Berbera - nel Nord - le installazioni navali sovietiche ${ }^{31}$.

L'URSS dopotutto aveva già iniziato ad avvicinarsi alla nuova Etiopia del Derg, che reclamava quanto e più di Siad Barre una vicinanza ideologica al modello politico e sociale sovietico e che era in quel momento in possesso dell'autorità sull'Ogaden nel quale il presidente somalo era nato.

\section{L'Ogaden}

La vasta regione dell'Ogaden, estendentesi per buona parte dell'attuale confine tre Etiopia e Somalia e già prima della fine della Seconda guerra mondiale popolata maggioritariamente da pastori nomadi e seminomadi di lingua somala, venne annessa all'Etiopia nel 1948. Nel 1960 si formò il JXSG (Jabhadda Xoreynta Somali Galbeed, Fronte di Liberazione Somalo Occidentale), il quale rivendicava alla Somalia l'Ogaden, sotto gli auspici della Lega dei Giovani Somali appena arrivata al governo che non mancò mai di finanziarlo e sostenerlo pubblicamente ${ }^{2}$. Il JXSG si rese protagonista dei primi scontri armati con l'esercito etiope nell'Ogaden, finché, nel 1964, non iniziò a intervenire anche l'esercito somalo in suo sostegno, portando lo scontro a un livello di guerra di confine tra le truppe armate che lo pattugliavano. Il rischio di una guerra totale rientrò pressoché immediatamente, e con l'avvento al potere di Siad Barre - che, ricordiamo, aveva preso il potere a scapito della Lega che era da sempre stata la prima

\footnotetext{
30 PATMAN, Robert G., op. cit., p. 117.

${ }^{31}$ ALLISON, Roy, The Soviet Union and the Strategy of Non-Alignment in the Third World, New York City, Cambridge University Press, 1988, p. 207.

32 KELLER, Edmond J., op. cit., p. 158.
} 
sostenitrice politica ed economica del JXSG - le tensioni sembrarono stemperarsi; nel 1973, però, vennero scoperti giacimenti di petrolio nella regione, e il livello di scontro si rialzò33. Siad Barre aveva già iniziato a promuovere gli elementi già citati di culto autocratico della personalità, e in un tale contesto promosse forme di nazionalismo a oltranza, che ebbero come prevedibile conseguenza la rivendicazione di territori abitati da somali: uno su tutti fu proprio l'Ogaden, in cui - nel villaggio di Shilaaba - era nato. La rivendicazione continuò anche con l'avvento al potere del non meno nazionalista Derg in Etiopia34, e sfruttando i suoi già citati conflitti interni nel febbraio 1977 il JXSG tornò ad attaccare con atti di guerriglia le truppe etiopi di stanza nella regione, tra le quali, per tutta risposta, iniziarono ammutinamenti di massa. La Somalia, vedendo la situazione propizia, a luglio inviò le proprie truppe al di là del confine, attaccando l'Etiopia. Era iniziata la guerra dell'Ogaden.

L'Etiopia, che già aveva aperto rapporti economici e militari con l'Unione Sovietica, ottenne i primi aiuti specifici per combattere l'invasione somala. Per tutta risposta la Somalia, che già aveva come detto iniziato ad aprire i propri porti meridionali alle forze navali statunitensi, interruppe ogni relazione con l'Unione Sovietica, ottenendo in cambio forniture di armi, supporto logistico e appoggio diplomatico dagli Stati Uniti.

Da parte statunitense il sostegno alla Somalia fu tanto economico e militare quanto politico. Ciononostante, il punto focale di concentrazione degli sforzi per l'ottenimento di alleanze nell'Africa Orientale, per gli Stati Uniti, era già stato l'Etiopia ${ }^{35}$. Ancora prima dello scoppio della guerra e tre anni dopo il colpo di Stato del Derg William Odom, assistente militare del National Security Council dell'appena eletto governo Carter, notava come l'importanza strategica dell'Etiopia dovesse essere considerata in un contesto più ampio, non limitato al resto del continente africano e considerante anche la vicinanza alla penisola arabica, e definiva la regione «un anello cruciale nella catena della difesa occidentale». Una volta perso il sostegno politico dell'Etiopia, che era il paese più esteso e popoloso della regione, la Somalia diventò in subordine il territorio su cui maggiormente dovessero concentrarsi gli sforzi statunitensi; questi, però, furono inferiori alle aspettative dello stesso Siad Barre, a causa anche del relativamente scarso avventurismo del governo Carter, il quale peraltro aveva impostato buona parte della propria autorappresentazione sulla cautela nell'assunzione

33 OFCANSKY, Thomas P., BERRY, LaVerle Bennette, Ethiopia: a country study, Washington, D.C., Library of Congress. Federal Research Division, 1993, p. 50.

34 CALCHI NOVATI, Giampaolo, Il Corno d'Africa nella storia e nella politica. Etiopia, Somalia e Eritrea fra nazionalismi, sottosviluppo e guerra, Torino, SEI, 1994, pp. 127-129.

35 CALCHI NOVATI, Giampaolo, «L'Imperatore e il Presidente: Alle Origini dell'Alleanza Etiopia - Stati Uniti», in Africa, 43, 3/1988, pp. 360-377; CARCANGIU, Bianca Maria, «Gli Stati Uniti e la questione dell'Ogaden (1950-1960)», in Africa, 52, 3/1997, pp. 365-399. 
di impegni militari internazionali ${ }^{36}$. Ogni invio di armi e supporto logistico considerato di carattere difensivo da parte statunitense cessò nel novembre 1977, quando la sconfitta somala sembrava inevitabile e la presidenza Carter aveva iniziato a muovere nel dibattito pubblico obiezioni sul carattere offensivo della guerra, al fine di giustificare il taglio delle forniture militari che sarebbero comunque riprese in seguito alla pacificazione37. Il sostegno saudita proseguì invece fino alla fine della guerra, conclusasi con il ritiro delle truppe somale dall'Ogaden il 9 marzo 1978.

Da parte del blocco sovietico, il sostegno all'Etiopia del Derg fu più diretto. Oltre al già citato invio di armi, logistica militare e finanziamenti, il generale Vasilij Petrov venne inviato come consulente militare sul campo per le forze etiopi e lavorò in piena cooperazione con il Derg38; l'Unione Sovietica fornì inoltre armamenti per un valore totale stimato intorno ai due milioni di dollari39. Il sostegno cubano fu ancora più netto. Sotto il comando del generale e comandante in capo dell'esercito cubano Arnaldo Ochoa, vennero inviate truppe per un totale compreso tra le 17000 e le 18000 unità40; nel 1982, a guerra ampiamente finita, rimanevano in Etiopia un totale di tredicimila effettivi cubani e 1350 consiglieri militari sovietici ${ }^{41}$.

Nonostante le narrazioni eurocentriche dominanti tendano a non riconoscere autonomia decisionale a paesi economicamente subalterni, comunque, la guerra dell'Ogaden non fu semplicemente una guerra per procura.

Somalia ed Etiopia erano entrambe paesi non allineati, sin da prima delle rispettive rivoluzioni. Il Movimento dei Paesi Non Allineati sin dalla sua nascita promosse un modello ideologico di distanza, di terzietà dai due blocchi del Nord del mondo in nome della decolonizzazione; per quanto riguardava l'ambito diplomatico, nei fatti, le posizioni e i rapporti con le dinamiche di potere dei due blocchi all'interno dei Non Allineati erano tra le più varie possibili, spaziando dalla vicinanza politica, commerciale e militare agli Stati Uniti all'ortodossia marxista. Etiopia e Somalia si limitarono, entrambe, a giocare la carta del non allineamento con maestria. Il Movimento dei Non Allineati era un comodo ombrello sotto il rifugio del quale si sarebbero potute ottenere le migliori condizioni economiche e politiche rivolgendosi ora a un blocco e ora all'altro

\footnotetext{
${ }^{36}$ JACKSON, Donna R., op. cit., p. 37.

37 SCHRAEDER, Peter J., United States Foreign Policy toward Africa. Incrementalism, Crisis and Change, New York City, Cambridge University Press, 1994, pp. 142, 148-150.

38 «Vasiliji Petrov», in Герой Советского Союза, URL: < http://www.warheroes.ru/hero/hero.asp?Hero_id=1937 > [consultato il 24 agosto 2015].

39 SCHRAEDER, Peter J., op. cit., p. 144.

40 RALSTON, Richard D., "Cuba in Africa and Africa in Cuba», in Contemporary Marxism, 7, 1983, p. 144.

${ }^{41}$ KATZ, Mark N., «The Soviet-Cuban Connection», in International Security, 8, 1/1983, pp. 9496.
} 
quando necessario. Entrambi i regimi, come tipico nel terzomondismo, portarono avanti retoriche di affermazione nazionale in indipendenza da qualunque istanza altra, presentando sé stessi e le proprie ideologie guida come prodotti nazionali.

C'erano state, inoltre, delle precedenti situazioni di tensione. Il confine dell'Ogaden venne stabilito dalla precedente amministrazione bellica britannica; sicuramente influì sul passaggio dell'amministrazione della regione all'Etiopia il fatto che il suo ritorno all'indipendenza con il ripristino della precedente autorità imperiale fosse avvenuto nel 1948, mentre la Somalia rimase divisa in protettorati ONU ad amministrazione fiduciaria italiana e britannica fino al 1960. Benché il concetto europeo di nazionalità possa avere un senso relativo quando applicato all'identificazione di comunità immaginate di contesti extraeuropei, una concezione di "nazione somala" era già abbondantemente visibile nella pratica politica di Maxamed Cabdille Xasan, leader dello Stato Derviscio che tra la fine del XIX secolo e l'inizio del XX lottò per vent'anni contro l'occupazione coloniale britannica e italiana. Xasan teorizzò per primo l'idea di una Grande Somalia, che nei suoi piani avrebbe dovuto essere uno Stato indipendente che raccogliesse qualunque territorio abitato da popolazione identificata come somala, comprendendo anche l'Ogaden ${ }^{42}$, e Siad Barre non mancò di perseguire gli stessi progetti territoriali. Xasan venne anzi più volte dipinto da lui stesso come un eroe nazionale, e con la campagna di alfabetizzazione venne operata una grande insistenza sull'insegnamento delle sue gesta, opportunamente mitizzate ${ }^{43}$. Già i predecessori di Siad Barre, peraltro, avevano considerato la rivendicazione dell'Ogaden come un fattore fondamentale ai fini della costruzione della nuova nazione somala, tanto da porre la promozione dell' «unione di tutti i territori somali» da parte del nuovo Stato nel quarto articolo della sua prima Costituzione, datata 196144.

L'Etiopia, invece, sin dall'epoca imperiale aveva entro i propri confini una gran varietà di gruppi distinguentisi l'uno dall'altro sotto il piano culturale. A tutt'oggi nessuna entità nazionale in Etiopia costituisce la maggioranza assoluta della popolazione: gli Oromo, il gruppo più numeroso, ne compongono solo il 34,49\%, seguiti dagli Amhara che ne compongono il 26,89\%45. La copiosa insistenza della

42 HESS, Robert L., «The 'Mad Mullah' and Northern Somalia», in The Journal of African History, 5, 3/1964, pp. 415-433.

43 BESTEMAN, Catherine, «Violent Politics and the Politics of Violence: The Dissolution of the Somali Nation-State», in American Ethnologist, 23, 3/1996, pp. 580-581, 588-591.

44 CALCHI NOVATI, Giampaolo, Il Corno d'Africa nella storia e nella politica, cit., p. 98.

45 Population and Housing Census Report - Country, 2007, pp. 73-74, in Central Statistical Agency, URL:

<http://www.csa.gov.et/newcsaweb/images/documents/surveys/Population\%20and\%20Housi ng\%20census/ETH-pop-2007/surveyo/data/Doc/Reports/National_Statistical.pdf

[consultato il 26 agosto 2015]. 
retorica politica del Derg sul nazionalismo etiope derivava dalla necessità di unire le differenti popolazioni di quello che era uno Stato plurinazionale nell'identificazione con l'entità statale e con i progetti politici del regime che la governava.

Di conseguenza la guerra dell'Ogaden fu anche uno scontro tra due nazionalismi, diversi nella teorizzazione in quanto rispondenti ai diversi contesti dai quali procedevano, ma aventi uno stesso obiettivo territoriale. Nello scontro tra tali nazionalismi si estrinsecò, in ultima istanza, lo scontro di due diverse concezioni di governo dittatoriale militare.

\section{Conclusioni}

Tra il governo autocratico di Siad Barre e la dittatura del Derg in Etiopia è facile trovare dei punti di continuità. Si è trattato in entrambi i casi di governi militari, guidati da figure provenienti dall'esercito. In entrambi i casi tali figure sono andate al potere attraverso colpi di Stato, nati per una comune insoddisfazione di militari ideologicamente vicini al socialismo e avvicinatisi a tale ideologia in risposta alle disuguaglianze economiche e sociali diffuse nei rispettivi paesi. Una volta al potere, entrambi i regimi utilizzarono metodi di governo dittatoriali, che prevedettero l'illegalizzazione delle opposizioni e l'utilizzo sistematico della violenza contro i loro esponenti, anche all'interno degli stessi organismi di governo, nonché lo stabilimento di un controllo politico serrato sulle forze armate che avevano portato entrambi i regimi al potere. In entrambi i casi, infine, vennero teorizzati sistemi ideologico-politici che prevedevano proprie letture della realtà, entrambi focalizzati sull'introduzione di pratiche di controllo economico ideologicamente ascrivibili al socialismo ed entrambi utilizzanti retorica nazionalista a vario titolo per l'affermazione delle proprie elaborazioni ideologiche nelle rispettive realtà sociali e culturali.

Soffermandoci sui punti di distacco tra l'autorità militare del Derg e quella di Siad Barre, quattro aspetti saltano all'occhio.

Innanzitutto va notata la differenza strutturale delle dinamiche decisionali. Il Derg, come il nome stesso suggerisce, era un consiglio che operava in totale collegialità, tanto da costringere Mengistu e la sua fazione alla sparatoria del 3 febbraio 1977 per conseguire una maggioranza stabile al suo interno. Anche con l'ottenimento di tale maggioranza, il Derg continuò ad agire con dinamiche consiliari, quantomeno nominalmente. Siad Barre, invece, ebbe sempre piena autonomia decisionale all'interno dell'organismo di governo, del quale ricopriva le cariche supreme sin dall'inizio della rivoluzione, avendo cariche di comando già da prima della presa di 
potere negli organismi militari dai quali partì il golpe.

Un altro fattore di differenziazione tra le due dittature militari fu senza dubbio la diversa metodologia di penetrazione dell'autoritarismo all'interno della società: il Derg creò uno Stato nuovo, controllando appieno i poteri locali attraverso le strutture militari che a esso facevano capo e rivoluzionando i rapporti di proprietà e di produzione. Siad Barre, al contrario, nonostante la promozione di un culto della personalità e più generalmente un maggiore sforzo di fidelizzazione della popolazione, non modificò in profondità i rapporti economici e sociali del territorio, pur con la nazionalizzazione delle imprese. Ciò è spiegabile con la differenza dei contesti di partenza: l'Etiopia pre-rivoluzione era un impero teocratico con un sistema di proprietà feudale, in mano ad aristocrazie di stampo nobiliare sistematizzate come tali da una forte autorità centrale. La Somalia, al contrario, aveva un'economia a maglie più larghe, nella quale i rapporti di potere economico e di autorevolezza politica erano controllati da un più liquido e mutevole sistema di clan.

In terzo luogo va ricordata la differenza ideologica, e in particolar modo le speculari presentazioni al pubblico dei principi del marxismo-leninismo e le altrettanto opposte aderenze effettive all'ortodossia da parte delle due dittature. Il Derg, pur presentando l'Hibret's'bawinet con una necessaria retorica nazionalista e tradizionalista e disdegnando ufficialmente qualunque apparato ideologico di organizzazione politica e sociale proveniente dall'estero in quanto tale, non esitò a riorganizzare la società etiope secondo principi politici ed economici marxisti. Siad Barre, invece, promosse ufficialmente il marxismo come base ideologica della nuova organizzazione politica ed economica della società somala, e di fatto produsse vari sforzi legislativi rispondenti ai suoi principi; a livello retorico, però, al marxismo-leninismo di matrice sovietica affiancò sempre, nell'indicazione dei principi di riferimento della nuova ideologia di Stato, i precetti dell'Islam. Siad Barre tese spesso a far leva sulla retorica islamica al fine di intavolare rapporti diplomatici con i ricchi Stati confessionali islamici della penisola araba, su tutti l'Arabia Saudita. Non può essere inoltre considerabile come totalmente compiuto il suo progetto di instaurazione di rapporti socioeconomici sulla base del marxismo-leninismo, per la sopra trattata preservazione dei preesistenti meccanismi di attività economica e controllo sociale a livello locale.

Allo stesso modo, infine, nacquero e si svilupparono specularmente le dinamiche di instaurazione di rapporti diplomatici con il paese il cui partito di governo rappresentava la guida politica, economica e culturale del marxismo-leninismo nel mondo contemporaneo: l'Unione Sovietica. Questa guardò con molto interesse alla Somalia, in cui il Siad Barre che aveva appena deposto il precedente regime si 
proclamava apertamente marxista. Siad Barre, però, fu come abbiamo visto restio a schierarsi nettamente con l'Unione Sovietica e a entrare nei suoi ambiti di clientelismo politico, che temeva potessero alienargli la possibilità di ottenere migliori condizioni di accordo economico e militare con il più geograficamente vicino mondo arabo. In Etiopia intanto la situazione si evolvette con il colpo di Stato del Derg, che, al contrario di Siad Barre, cercò immediatamente la vicinanza politica con l’URSS, pagando tale presa di posizione con la fine degli aiuti statunitensi. Tale vicinanza venne incontrata senza eccessive resistenze da parte sovietica, facilitata anche dalle manovre di avvicinamento politico, economico e militare di Siad Barre ai paesi arabi al di là del Golfo Arabico e agli Stati Uniti a cui facevano riferimento. 


\section{* L'autore}

Francesco Maria Mengo, ha conseguito la Laurea presso l'Università degli Studi di Macerata. Al momento è dottorando in Storia dell’Universitat Pompeu Fabra di Barcellona.

URL: < http://www.studistorici.com/progett/autori/\#Mengo >

\section{Per citare questo articolo:}

MENGO, Francesco Maria, «ll Derg, Siad Barre e l'Ogaden. Due diverse concezioni di dittatura militare e il loro scontro in una guerra per procura», Diacronie. Studi di Storia Contemporanea : Le dittature militari: fisionomia ed eredità politica, 29/12/2015,

URL:< http://www.studistorici.com/2015/12/29/mengo_numero_24/ >

Diacronie Studi di Storia Contemporanea 0 www.diacronie.it

Risorsa digitale indipendente a carattere storiografico. Uscita trimestrale.

redazione.diacronie@hotmail.it

Comitato di redazione: Jacopo Bassi - Luca Bufarale - Elisa Grandi - Antonio César Moreno Cantano - Deborah Paci - Fausto Pietrancosta - Alessandro Salvador - Matteo Tomasoni - Luca Zuccolo

Diritti: gli articoli di Diacronie. Studi di Storia Contemporanea sono pubblicati sotto licenza Creative Commons 3.0. Possono essere riprodotti e modificati a patto di indicare eventuali modifiche dei contenuti, di riconoscere la paternità dell'opera e di condividerla allo stesso modo. La citazione di estratti è comunque sempre autorizzata, nei limiti previsti dalla legge. 